This document is the accepted manuscript version of the following article:

Donnel1y, C., Finizio, S., Gliga, S., Holler, M., Hrabec, A., odstrčil, M., ... Raabe, J. (2020). Time-resolved imaging of three-dimensional nanoscale magnetization dynamics. Nature Nanotechnology. https://doi.org/10.1038/s41565-020-0649-x

\title{
Time-resolved imaging of three-dimensional nanoscale magnetization dynamics
}

Authors: Claire Donnelly ${ }^{1,2,3^{*}}$, Simone Finizio², Sebastian Gliga ${ }^{2,3,4}$, Mirko Holler², Aleš Hrabec ${ }^{2,3,5}$, Michal Odstrčil2, Sina Mayr ${ }^{2,3}$, Valerio Scagnoli ${ }^{2,3}$, Laura J. Heyderman ${ }^{2,3}$, Manuel Guizar-Sicairos ${ }^{2 *}$, Jörg Raabe ${ }^{2^{*}}$.

Affiliations:

${ }^{1}$ Cavendish Laboratory, University of Cambridge, JJ Thomson Ave, Cambridge, CB3 OHE, UK.

2 Paul Scherrer Institute, 5232 Villigen, Switzerland.

${ }^{3}$ Laboratory for Mesoscopic Systems, Department of Materials, ETH Zurich, 8093 Zurich, Switzerland.

${ }^{4}$ SUPA, School of Physics and Astronomy, University of Glasgow, Glasgow G12 8QQ, UK.

${ }^{5}$ Laboratory for Magnetism and Interface Physics, Department of Materials, ETH Zurich, 8093

Zurich, Switzerland

*Correspondence to: cd691@cam.ac.uk, manuel.guizar-sicairos@psi.ch, joerg.raabe@psi.ch.

One Sentence Summary: Knowledge and control of the magnetization dynamics in micromagnetic configurations is important both from a fundamental point of view, and for technological applications. Pump-probe magnetic laminography now unveils the magnetization dynamics of a three-dimensional system with nanoscale resolution.

Main Text:

Understanding and controlling the dynamic response of magnetic materials with a threedimensional magnetization distribution is important both fundamentally and for technological applications. From a fundamental point of view, the internal magnetic structure and dynamics in known bulk materials still need to be mapped [1], including the dynamic properties of topological structures such as vortices [2], magnetic singularities [3] or skyrmion lattices [4]. From a technological point of view, the response of inductive materials to magnetic fields and spin-polarized currents is essential for magnetic sensors and data storage devices [5]. Here, we demonstrate time-resolved magnetic laminography, a pump-probe technique, which offers access to the temporal evolution of a complex three-dimensional magnetic microdisc with nanoscale resolution, and with a synchrotron-limited temporal resolution of $70 \mathrm{ps}$. We image the dynamic response to a $500 \mathrm{MHz}$ magnetic field of the complex three-dimensional magnetization in a two-phase bulk magnet with a lateral spatial resolution of $50 \mathrm{~nm}$. This is achieved with a stroboscopic measurement consisting of 8 time steps evenly spaced over $2 \mathrm{~ns}$. These measurements map the spatial transition between domain wall motion and the dynamics of a uniform magnetic domain that is attributed to variations in the magnetization state across the phase boundary. The capability to probe three-dimensional magnetic structures with temporal resolution paves the way for the experimental investigation of novel functionalities arising from dynamic phenomena in bulk and three-dimensional patterned nanomagnets [6]. 
While magnetization dynamics have been measured in 2D structures, and are well understood for isolated magnetic structures in thin films, such as vortices [7-10], domain walls [11-14] and skyrmions $[15,16]$, until now insight into three-dimensional dynamics has only been possible through comparison with simulations [17]. Static three-dimensional magnetic imaging has recently been achieved using hard X-ray [18, 19], neutron [20-22], electron [23] and soft X-ray $[24,25]$ tomographies which provide a means to determine the three-dimensional magnetic configurations. Of these methods, synchrotron X-rays are unique owing to their ability to provide high spatial resolution imaging of thick materials $[18,26]$, as well as element-specific information. Additionally, the pulsed time structure of synchrotron X-rays naturally provides the possibility to perform time-resolved measurements: pump-probe measurements of material systems have proved to be a powerful tool for the measurement of $\mathrm{MHz}$ and $\mathrm{GHz}$ dynamics within a range of magnetic materials and systems [27]. Such time-resolved measurements generally require flat samples, which pose challenges when it comes to threedimensional tomographic magnetic imaging. In particular, there is a "missing wedge" due to high absorption of the sample at high angles, and it is necessary to alter the orientation of the sample with respect to the rotation axis part way through the measurement in order to measure all three components of the magnetization. As a result, until now it has not been possible to combine three-dimensional magnetic imaging with dynamic measurements.

The imaging of magnetization dynamics in three-dimensions becomes much more feasible with an alternative geometry for 3D imaging, laminography [28]. For magnetic imaging, as shown in this work, laminography provides immediate access to all three components of the magnetization with a single axis of rotation and, by combining vectorial magnetic imaging with a pump-probe setup, it is possible to obtain the temporal evolution of the entire threedimensional vector field. In this way, we can study the surface excitations as well as the dynamics of buried three-dimensional magnetic domains and domain walls.

Here, we image the as-grown three-dimensional magnetic state of a GdCo microdisc with a diameter of $5 \mu \mathrm{m}$ and a thickness of $1.2 \mu \mathrm{m}$ using magnetic laminography, and determine its dynamic response to a radio frequency (RF) magnetic field. The microdisc was patterned by milling a continuous film deposited on the back of a $\mathrm{Si}_{3} \mathrm{~N}_{4}$ membrane with a focused ion beam [see inset of Fig. 1a, ii for scanning electron micrograph of the sample]. A two-phase GdCo film was deposited by magnetron sputtering, first with the sample continuously rotating for the lower part, and then stationary for the top 1/4 of the thickness. On the front of the membrane a $300 \mathrm{~nm}$-thick Cu stripline was patterned, through which an RF current was injected to produce an in-plane magnetic field excitation.

The experimental setup is shown schematically in Figure 1a, where the plane of the sample is tilted at an angle of $29^{\circ}$ to the propagation direction of the X-rays. During the measurement the sample is rotated about an axis perpendicular to its surface plane, and two-dimensional projections of the magnetic microdisc are measured with dichroic ptychography [26]. Twodimensional projections of the magnetic configuration of the GdCo microdisc for the sample rotated by $0^{\circ}$ and $45^{\circ}$ about the laminography rotation axis are shown in Figure $1 \mathrm{c}$ and $1 \mathrm{~d}$, respectively. For these projections, a multi-domain state can be identified at the bottom of the structure, that appears to evolve through the thickness of the structure. 

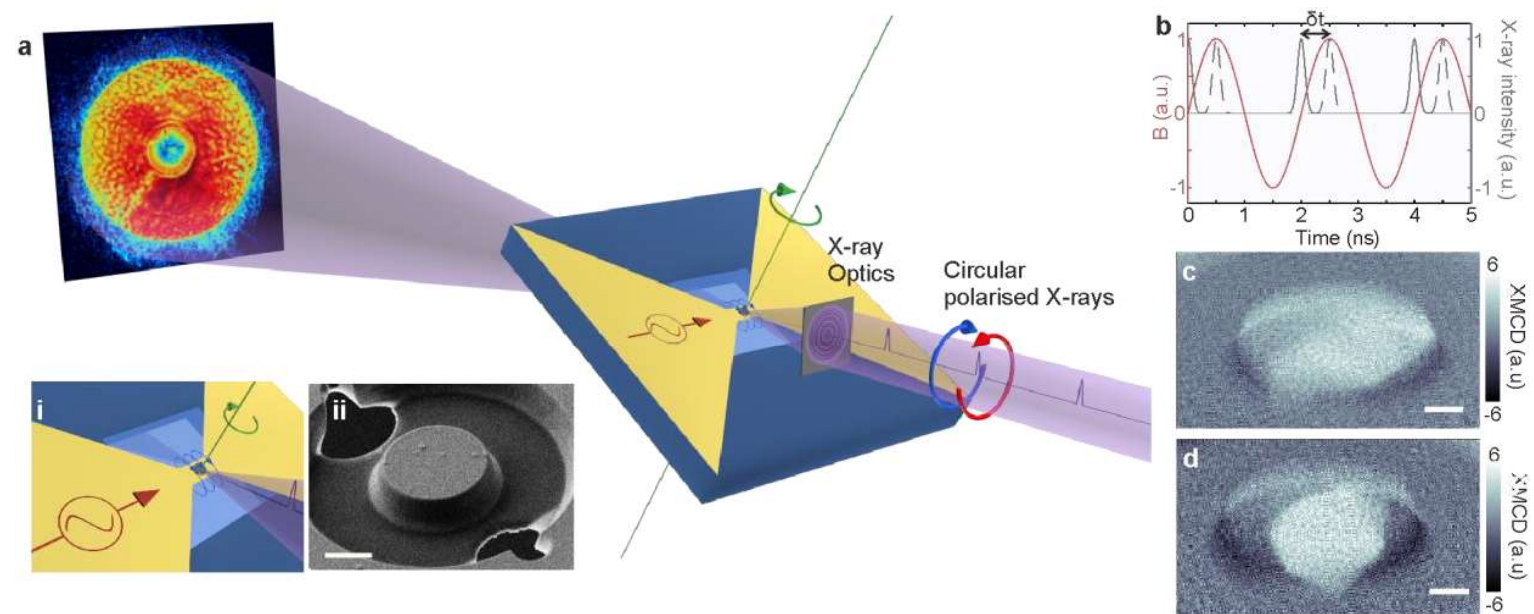

Figure 1. Time-resolved X-ray magnetic laminography. a) The experimental setup. For synchrotron X-ray magnetic laminography, ptychographic projections are measured with right- and left-handed circular polarization with the sample rotated around an axis that is at $61^{\circ}$ with respect to the $\mathrm{X}$-ray beam propagation direction. To observe the magnetization dynamics, the sample is fabricated on a Cu stripline (see magnified view in $\mathrm{i}$, along with a scanning electron micrograph of the sample in ii), and excited with a $500 \mathrm{MHz}$ RF magnetic field produced by passing a current through the stripline. Magnetic laminography is then performed for a number of different delay times of the RF magnetic field with respect to the X-ray pulses. b) the concept of pump-probe magnetic laminography is illustrated, where the RF magnetic field excitation (red oscillatory waveform) is frequency and phase matched to the incoming X-ray pulses (purple waveforms). By varying the time delay $\delta t$ between the continuous RF magnetic field excitation (red) and the X-ray bunches (two temporal measurements, separated by $\delta \mathrm{t}$, are indicated by the full and dashed purple waveforms), and repeating the laminography measurement for different $\delta \mathrm{t}$, temporally-resolved maps of the magnetization vector field are obtained. XMCD projections of the magnetic microdisc are shown in c) and d) for the sample at $0^{\circ}$ and $45^{\circ}$, respectively. Scale bars represent $2 \mu \mathrm{m}$ in a,ii and $1 \mu \mathrm{m}$ in c,d.

For magnetic laminography, 2D ptychographic projections were measured at 144 angles equally distributed over $360^{\circ}$ with both right and left circularly polarized X-rays. The three-dimensional magnetic configuration was reconstructed using a graphics processing unit (GPU)

implementation of the gradient-based arbitrary projection magnetic reconstruction algorithm, detailed in [29], with a lateral spatial resolution of approximately $50 \mathrm{~nm}$. The RF magnetic field used to excite the sample was frequency and phase-matched to the synchrotron X-ray pulses, which are 70 ps wide $X$-ray flashes occurring every $2 \mathrm{~ns}$ (repetition frequency of $500 \mathrm{MHz}$ ). To probe the dynamic response of the sample, a magnetic laminography dataset was acquired for a number of different delay times of the excitation field with respect to the X-ray bunches, as shown schematically in Figure $1 b$.

The reconstructed three-dimensional magnetization distribution of the GdCo microdisc for zero delay time between the excitation and the X-ray bunches is shown in Figure 2 . In the lower region of the structure, a multi-domain state is present, in which the domains are separated by elongated vortex domain walls (Figure 2a). Regarding the evolution of the magnetic configuration through the disk thickness, the central domain expands and the vortex domain walls are located increasingly closer to the edges of the sample (Figure $2 \mathrm{~b}$ ), until they are expelled approximately mid-way through the sample, resulting in a single magnetic domain with a curling magnetization at the edges, forming an S-state (Figure 2c). At the top of the microdisc, the magnetization forms an almost uniform state (Figure $2 \mathrm{~d}$ ). The positions of the vortex cores are plotted using isosurfaces corresponding to a minimum in-plane magnetisation in Figure 2e. Within the core of a vortex, the magnetization points out of the plane of the disk, 
and the direction of the magnetization (i.e. $\pm m_{y}$ ), defines its polarization. Here, the isosurfaces representing the core of the vortices are colored by the $m_{y}$ component, which can be seen to be positive (orange) and negative (green), indicating that the pair of vortices have opposite polarizations.

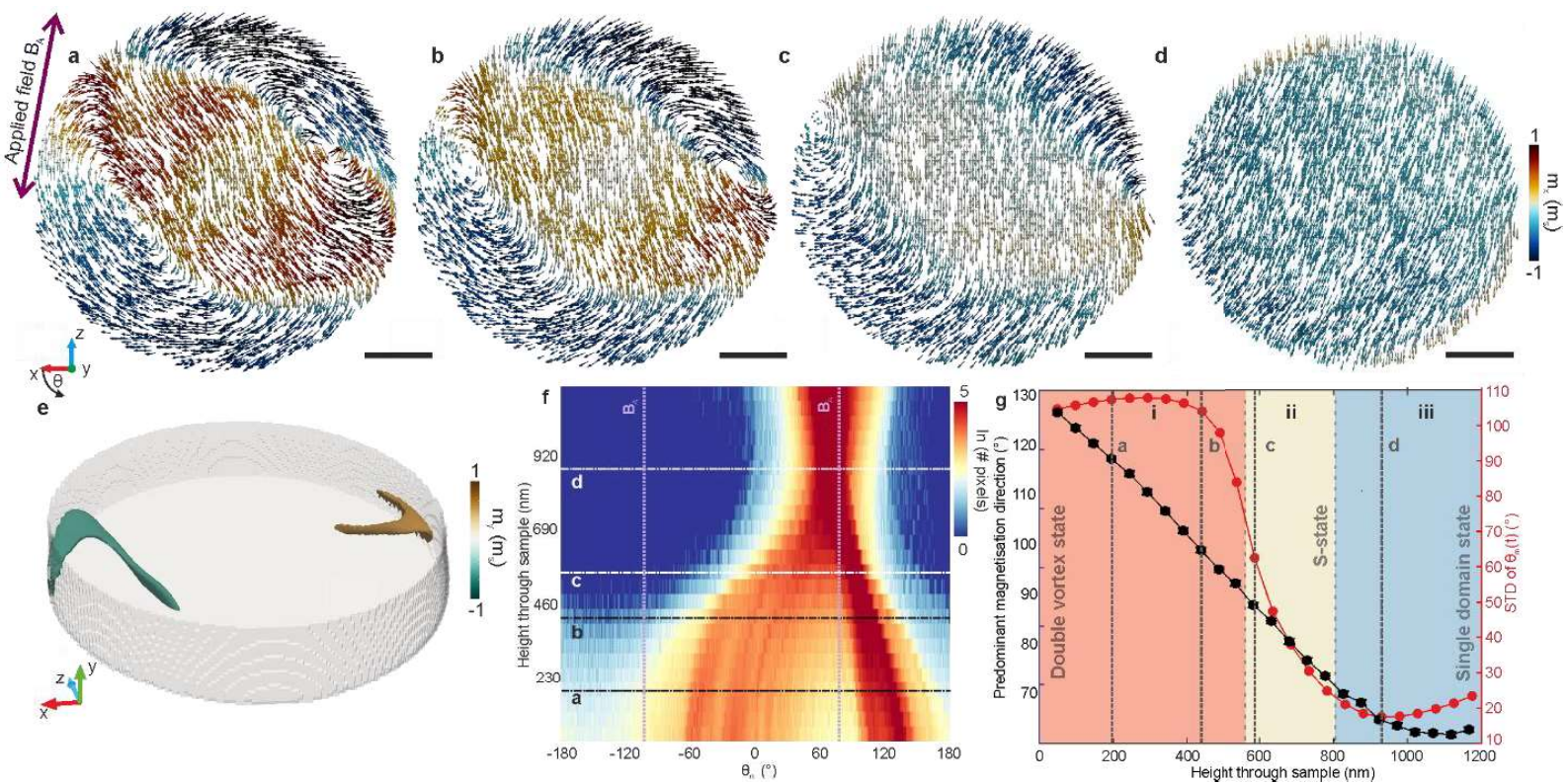

Figure 2 The reconstructed three-dimensional magnetic configuration of the GdCo microdisc for zero time delay between the Xray measurements and the RF excitation field $B_{A}$. At the bottom of the disc (a), a multidomain state forms with a large central domain and two vortices. With increasing height, the vortices move towards the edges of the disc (b), until they exit the structure approximately midway through the disc thickness, resulting in a large magnetic domain in an S-state (c). At the top of the disc, the magnetic configuration is almost uniform (d). The positions of the cores of the vortices, coloured by the ycomponent of the magnetization, are shown in (e), indicating that the magnetization in the cores of the two vortices have opposite directions, i.e. opposite vortex polarizations. These vortices eventually exit through the edges of the sample approximately mid-way through its height. f) Bivariate histogram showing the number of pixels as a function of magnetization direction (angle defined with coordinate axes in a) and thickness through the sample, with the orientation of the applied RF field $B_{A}\left[\theta=79^{\circ}\left(259^{\circ}\right)\right]$ indicated with purple dashed lines. $g$ ) The dominant direction of the magnetization in the central domain (black dots) and the standard deviation of $\Theta_{m}$ (red dots), which reflects the magnetization state, are given as a function of position through the thickness of the sample. Three regions of the sample are identified on the plot, the vortex state (i, orange), the S-state (ii, yellow) and the single domain state (iii, blue).

The gradual transition between the multi-domain state in the lower region of the sample to the single domain state in the upper region is attributed to variations in the anisotropy of the system. The rotation of the sample during sputtering led to effectively negligible anisotropy in the bottom part of the disc such that the magnetic state is predominantly determined by the magnetostatic energy. In contrast, the absence of rotation during the latter part of the deposition gave rise to the development of a sizeable uniaxial anisotropy in the top part of the sample. Therefore, the resulting system can be understood as a two phase system with a soft (bottom) and a hard (top) phase, conceptually analogous to an exchange-spring system [30].

We highlight the distribution of the direction of the magnetization through the thickness of the sample in Figure $2 \mathrm{f}$ in a bivariate histogram. At the bottom of the film, there is a large spread in the magnetization directions, which is consistent with the presence of the vortex state, with a pronounced peak centered around $140^{\circ}$ corresponding to the dominant direction of the 
magnetization in the central domain. With increasing height, the spread in directions is reduced, giving rise to a single peak corresponding to the narrower range of magnetization directions in the top domain. The standard deviation of the magnetization, which reflects the distribution of the magnetization directions in each state, is plotted as a function of thickness through the sample in Figure $2 \mathrm{~g}$ (red dots), and three main regions can be identified: the vortex state (i), the single domain state (iii), and an intermediate region corresponding to the curled $\mathrm{S}$ state (ii). The dominant direction of the magnetization can be tracked through the thickness of the sample by identifying the corresponding peak in the histogram for each slice in Figure $2 \mathrm{~g}$ (black dots). This magnetization direction varies linearly with distance through the sample up to a height of $900 \mathrm{~nm}$, after which the magnetization direction remains almost constant. This linear rotation of the magnetization direction along the thickness correlates with the position of the vortex domain walls. Indeed, as the thickness increases and the effective magnetic anisotropy increases, the vortex cores (plotted using isosurfaces in Fig 2e) are located increasingly closer to the edges of the sample and eventually expelled with the onset of strong uniaxial anisotropy in the top part of the disk.

We now probe the magnetization dynamics in response to the RF magnetic field of amplitude approximately $4 \mathrm{mT}$, orientated along the direction shown in Figure $2 \mathrm{a}\left[\theta=79^{\circ}\left(259^{\circ}\right)\right]$, by performing magnetic laminography for seven delay times over a period of 2 ns with respect to the $\mathrm{X}$-ray pulse train.
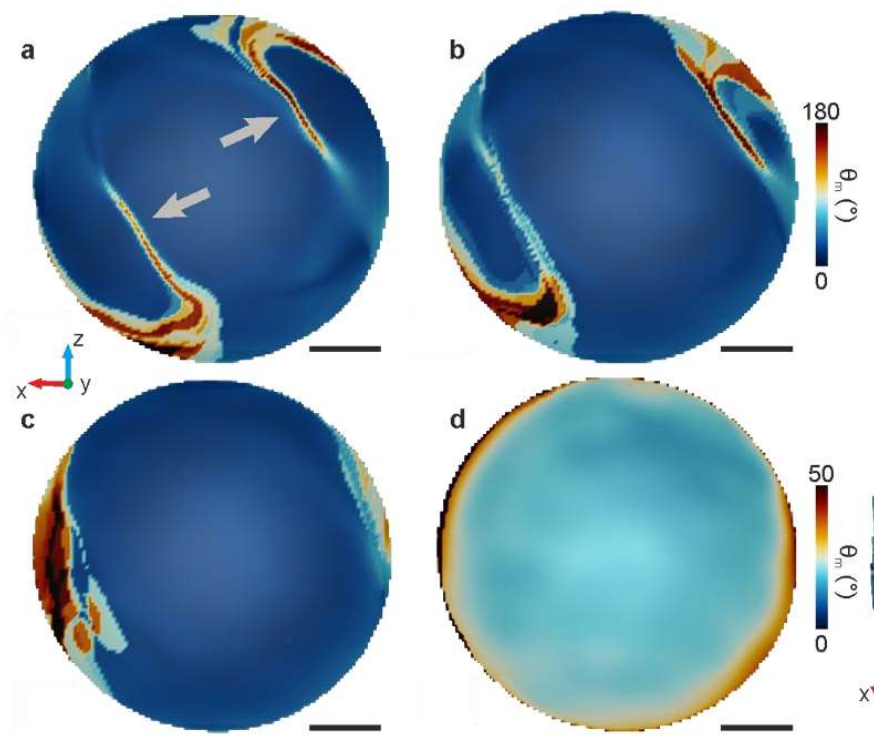

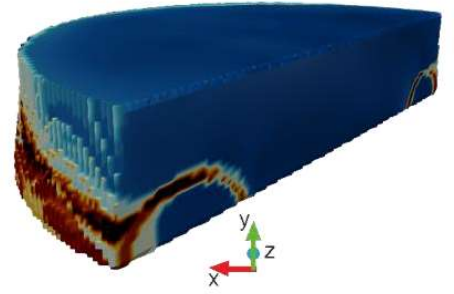

f

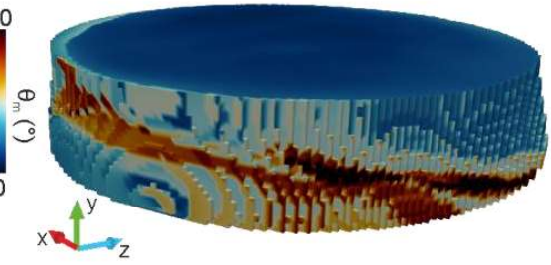

Figure 3. Dynamic response of the three-dimensional magnetization at $500 \mathrm{MHz}$. a-d) The precession angle of the magnetization is plotted for the heights within the sample corresponding to Fig 2 a-d (see Fig. S4 for slice locations). In the lower part of the sample $(a, b)$, there is significant precession in the vicinity of the vortex domain walls (indicated with grey arrows in a), as well as at the edge of the sample. In the intermediate S-state (c), an enhancement of the edge modes at opposite sides of the disc is observed. In the single domain state (d), lower amplitude edge modes are present on the top left and bottom right edges of the microdisc (visualised with a colour scale that is different to that used for a-c,e,f). The threedimensional dynamics are visualised in e and $\mathrm{f}$ by mapping the magnetisation precession: the dynamics are limited to the vortex cores (e) and edge modes, which spiral around the disc (f). Scale bars represent $1 \mu \mathrm{m}$.

We map the spatial distribution of the magnetization dynamics by plotting the Fourier coefficient of the temporal evolution of the in-plane magnetization direction $\theta_{m}$ corresponding 
to $500 \mathrm{MHz}$, in Figure 3. The precession angle of the magnetization is plotted in Figure 3a-d for the four magnetic states shown in Figure 2a-d, respectively. Magnetization dynamics are observed in the whole of the sample as a result of the inhomogeneous, coupled magnetization structure, although the field-induced torque is minimal in the top region of the sample (Figure $3 \mathrm{~d}$ ) because the direction of the applied RF magnetic field is almost parallel to the direction of the magnetization. At the bottom of the sample (Fig. 3 a,b - region (i)) the magnetization oscillates in the vicinity of the vortex walls indicating their reversible displacement (red elongated regions, indicated by grey arrows in Fig. 3a). The magnetization also oscillates at the bottom left and top right edges of the disc, where the magnetization is almost perpendicular to the applied RF field, thereby maximizing the field-induced torque. For the intermediate S-state (region (ii)), magnetization precession is observed in two regions close to the sample edge, which are adjacent to the location where the vortices are expelled from the disc (Figure 3c). In the single domain state in the upper part of the disc (Figure $3 \mathrm{~d}$ ), oscillations of the magnetization in the bulk of the domain are mostly absent (only low angle oscillations are present owing to the slightly inhomogeneous structure of the single domain state), and lower amplitude edge modes are present, made visible by associating the same colour bar with a smaller range of $\left[0-50^{\circ}\right]$. Three-dimensional maps of the modes are given in Figure $3 e, f$, showing that the magnetization dynamics are limited to the vortex domain walls and the edges of the sample. Mapping the magnetization dynamics provides insight into the interaction between the different domain structures and the resulting modifications in their dynamics. Moving up through the sample thickness, the edge oscillations are located at different positions, describing a spiral, which follows the evolution of the location of the vortex walls. The presence of vortices gives rise to regions with a sizeable magnetization component perpendicular to the applied field direction, where the field-induced torque is larger. In addition, the large anisotropy in the top region of the sample would be expected to lead to the formation of an 'onion' state [31] characterized by edge modes at the extremities of the domain, where the magnetization typically is not parallel to the sample boundaries. In contrast, in our system, imprinting of the vortices in the lower region onto the magnetization of the top region rather leads to the formation of a flower state [32], thus shifting the edge regions in which the magnetization oscillates around the edge of the disc.

Having identified the regions excited by the applied magnetic field, we now consider the details of the temporal evolution of the vortex domain walls in the lower section of the disc in Figure 4. The curl of the in-plane magnetization $\nabla \times m_{x z}$ is plotted for the slice at height $h=90 \mathrm{~nm}$ in Figure $4 a$, where the red and blue stripes correspond to the two vortex walls with opposite vorticities. In the insets, $\nabla \times m_{x z}$ is plotted in the vicinity of the domain wall with positive vorticity (region indicated by the black dashed box) for different time delays spanning a period of $2 \mathrm{~ns}$. Over this period, the domain wall moves about the reference position (dashed line) and its displacement in the direction perpendicular to the long axis of the wall is plotted as a function of time for $h=46 \mathrm{~nm}$ in Figure $4 \mathrm{~b}$, and for all sample heights in Extended Data 6, where the vortex domain wall can be seen to consistently exhibit an oscillatory motion. The amplitude of the domain wall oscillations is determined by calculating the Fourier coefficient of the temporal domain wall displacement. Within the lower $500 \mathrm{~nm}$ of the sample the amplitude 
is $74 \pm 4 \mathrm{~nm}$, with a maximum domain wall displacement of $200 \pm 15 \mathrm{~nm}$ for $h=46 \mathrm{~nm}$, corresponding to a domain wall velocity of approximately $200 \mathrm{~m} / \mathrm{s}$.

The domain wall with negative vorticity (blue in Figure 4a) displays a similar oscillatory motion perpendicular to its long axis with an amplitude of $(45 \pm 13) \mathrm{nm}$. Plotting the displacement of the domain walls as a function of time (Figure $4 \mathrm{~b}$ ) reveals that the domain walls oscillate out of phase. We quantify the phase difference between the two domain walls by calculating the phase of the Fourier coefficients, and find it to be $\frac{3 \pi}{4} \pm \frac{\pi}{4}$, equivalent to a delay of $0.75 \pm$ $0.25 \mathrm{~ns}$, essentially meaning that the domain walls essentially move in phase, but in opposite directions with the error resulting from a variation in the phase through the thickness of the sample. This motion corresponds to the breathing of the central domain, which is driven by the precessional dynamics of the oppositely-polarised vortex walls [9].

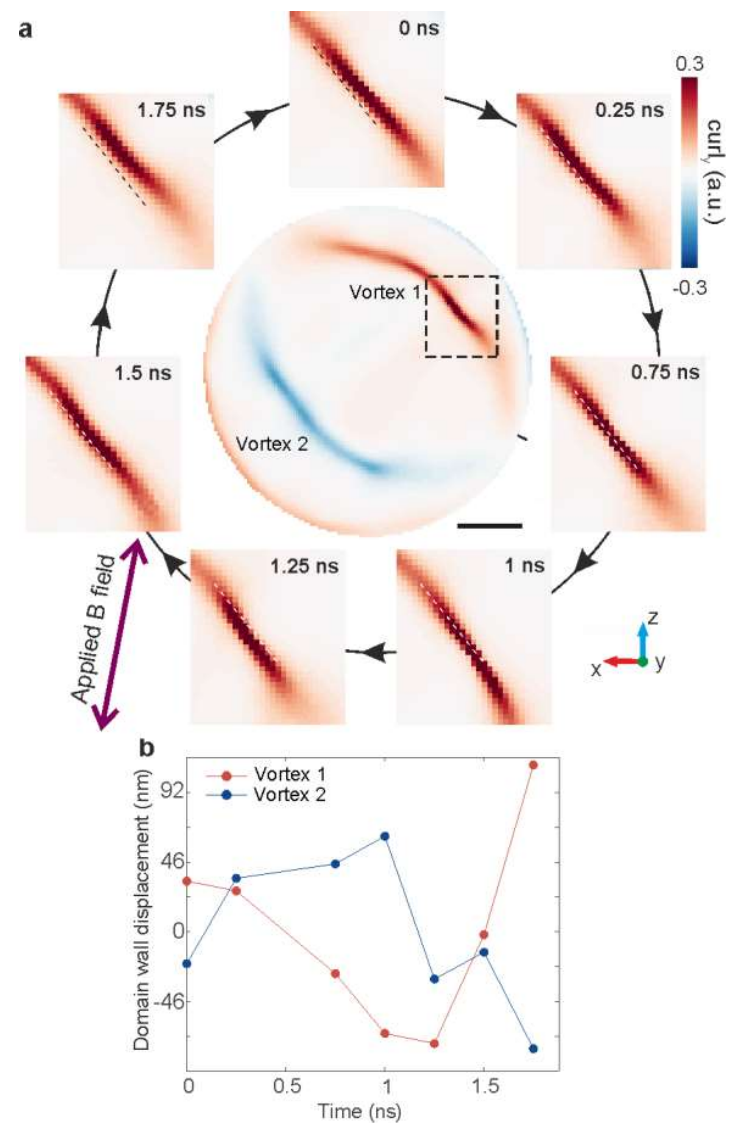

Figure 4. Domain wall dynamics in the lower region of the disc containing two vortex walls. a) The curl of the in-plane magnetization $\nabla \times m_{x z}$ for $\mathrm{h}=90 \mathrm{~nm}$ is shown, where the positions of the two domain walls are identified by the positive (red) and negative (blue) lines. The domain wall with positive curl in the region indicated by the dashed box is shown at different times increasing clockwise around the central image, with a reference location indicated by a dashed line. b) The dynamics of the wall with a positive curl vortex (red, vortex 1 ) are compared with the dynamics of the wall with a negative curl vortex (blue, vortex 2) for an example slice of height $46 \mathrm{~nm}$. The two oscillatory signals are out of phase by $\frac{3 \pi}{4} \pm \frac{\pi}{4},(0.75 \pm 0.25) \mathrm{ns}$, consistent with the breathing of the central domain with the applied RF magnetic field. Scale bar represents $1 \mu \mathrm{m}$. 
By combining magnetic laminography with a pump-probe experimental setup, we have imaged the magnetization dynamics of a three-dimensional magnetic microdisc in response to the application of an RF magnetic field. Two main types of magnetization dynamics are identified: the breathing mode of a central magnetic domain involving domain wall motion and magnetization dynamics at the edges of a uniform magnetic domain, that are enhanced by variations in the magnetization state across the phase boundary. At the boundary of the two phases defined by different magnetic anisotropies, the onset of a strong magnetic anisotropy in the upper region leads to the expulsion of the domain walls and the enhancement of edge mode magnetization dynamics. Given that laminography is ideal for the study of flat and thin samples, pump-probe laminography is directly compatible with high spatial resolution soft Xray imaging, making possible the direct application of the technique to nanoscale systems. This time-resolved laminography measurement took approximately 100 hours, or four days: with the significant increase in flux that comes with the next generation of synchrotrons, the time required for these measurements will decrease dramatically, making the three-dimensional mapping of nanoscale dynamics within a wider variety of systems feasible within the constraints of synchrotron beamtime. The ability to experimentally measure complex magnetization dynamics in three-dimensional systems is key for the investigation of the dynamic properties of 3D systems that have been proposed for future data storage [33], logic [34] and microwave [35, 36] applications.

\section{References:}

1. Templeton, T.L., Hanham, S.D. and Arrott, A.S., Helical patterns of magnetization and magnetic charge density in iron whiskers. AIP Advances, 2018. 8(5): p. 056022.

2. Yan, M., Hertel, R. and Schneider, C.M., Calculations of three-dimensional magnetic normal modes in mesoscopic permalloy prisms with vortex structure. Physical Review B, 2007. 76(9): p. 094407.

3. Im, M.-Y., et al., Dynamics of the Bloch point in an asymmetric permalloy disk. Nature Communications, 2019. 10(1): p. 593.

4. Milde, P., et al., Unwinding of a Skyrmion Lattice by Magnetic Monopoles. Science, 2013. 340(6136): p. 1076.

5. Hubert, A. and Schäfer, R., Magnetic Domains: The Analysis of Magnetic Microstructures. 1998: Springer.

6. $\quad$ Fernández-Pacheco, A., et al., Three-dimensional nanomagnetism. Nature Communications, 2017. 8: p. 15756.

7. $\quad$ Raabe, J., et al., Quantitative Analysis of Magnetic Excitations in Landau Flux-Closure Structures Using Synchrotron-Radiation Microscopy. Physical Review Letters, 2005. 94(21): p. 217204.

8. Wintz, S., et al., Magnetic vortex cores as tunable spin-wave emitters. Nature Nanotechnology, 2016. 11: p. 948.

9. $\quad$ Choe, S.B., et al., Vortex Core-Driven Magnetization Dynamics. Science, 2004. 304(5669): p. 420.

10. Van Waeyenberge, B., et al., Magnetic vortex core reversal by excitation with short bursts of an alternating field. Nature, 2006. 444(7118): p. 461-464.

11. Baumgartner, M., et al., Spatially and time-resolved magnetization dynamics driven by spin-orbit torques. Nature Nanotechnology, 2017. 12: p. 980.

12. Finizio, S., et al., Dynamic Imaging of the Delay-and Tilt-Free Motion of Néel Domain Walls in Perpendicularly Magnetized Superlattices. Nano Letters, 2019. 19(1): p. 375-380.

13. Stein, F.-U., et al., Time-resolved imaging of nonlinear magnetic domain-wall dynamics in ferromagnetic nanowires. Scientific Reports, 2013. 3: p. 1737.

14. Vogel, J., et al., Direct Observation of Massless Domain Wall Dynamics in Nanostripes with Perpendicular Magnetic Anisotropy. Physical Review Letters, 2012. 108(24): p. 247202.

15. Woo, S., et al., Deterministic creation and deletion of a single magnetic skyrmion observed by direct timeresolved X-ray microscopy. Nature Electronics, 2018. 1(5): p. 288-296.

16. Büttner, F., et al., Dynamics and inertia of skyrmionic spin structures. Nature Physics, 2015. 11: p. 225. 
17. Wartelle, A., et al., Bloch-point-mediated topological transformations of magnetic domain walls in cylindrical nanowires. Physical Review B, 2019. 99(2): p. 024433.

18. Donnelly, C., et al., Three-dimensional magnetization structures revealed with X-ray vector nanotomography. Nature, 2017. 547: p. 328.

19. Suzuki, M., et al., Three-dimensional visualization of magnetic domain structure with strong uniaxial anisotropy via scanning hard X-ray microtomography. Applied Physics Express, 2018. 11(3): p. 036601.

20. Hilger, A., et al., Tensorial neutron tomography of three-dimensional magnetic vector fields in bulk materials. Nature Communications, 2018. 9(1): p. 4023.

21. Kardjilov, N., et al., Three-dimensional imaging of magnetic fields with polarized neutrons. Nature Physics, 2008. 4(5): p. 399-403.

22. Manke, I., et al., Three-dimensional imaging of magnetic domains. Nat Commun, 2010. 1: p. 125.

23. Tanigaki, T., et al., Three-Dimensional Observation of Magnetic Vortex Cores in Stacked Ferromagnetic Discs. Nano Letters, 2015. 15(2): p. 1309-1314.

24. Streubel, R., et al., Retrieving spin textures on curved magnetic thin films with full-field soft X-ray microscopies. Nat Commun, 2015. 6: p. 7612.

25. Blanco-Roldan, C., et al., Nanoscale imaging of buried topological defects with quantitative X-ray magnetic microscopy. Nat Commun, 2015. 6: p. 8196.

26. Donnelly, C., et al., High-resolution hard $x$-ray magnetic imaging with dichroic ptychography. Physical Review B, 2016. 94(6): p. 064421.

27. Magnetism and Synchrotron Radiation, ed. E. Beaurepaire, et al. 2010: Springer.

28. Holler, M., et al., Three-dimensional imaging of integrated circuits with macro- to nanoscale zoom. Nature Electronics, 2019. 2(10): p. 464-470.

29. Donnelly, C., et al., Tomographic reconstruction of a three-dimensional magnetization vector field. New Journal of Physics, 2018. 20(8): p. 083009.

30. Kneller, E.F. and Hawig, R., The exchange-spring magnet: a new material principle for permanent magnets. IEEE Transactions on Magnetics, 1991. 27(4): p. 3588-3560.

31. Ha, J.K., Hertel, R. and Kirschner, J., Micromagnetic study of magnetic configurations in submicron permalloy disks. Physical Review B, 2003. 67(22): p. 224432.

32. Gatel, C., et al., Size-Specific Spin Configurations in Single Iron Nanomagnet: From Flower to Exotic Vortices. Nano Letters, 2015. 15(10): p. 6952-6957.

33. Parkin, S.S.P., Hayashi, M. and Thomas, L., Magnetic domain-wall racetrack memory. Science, 2008. 320(5873): p. 190-194.

34. Allwood, D.A., et al., Magnetic Domain-Wall Logic. Science, 2005. 309(5741): p. 1688-1692.

35. Kaka, S., et al., Mutual phase-locking of microwave spin torque nano-oscillators. Nature, 2005. 437(7057): p. 389-392.

36. Mancoff, F.B., et al., Phase-locking in double-point-contact spin-transfer devices. Nature, 2005. 437(7057): p. 393-395.

\section{Acknowledgments:}

The authors would like to thank Dario Marty for initial discussions about the sample fabrication and design, Elisabeth Müller for FIB patterning of the sample. All data was measured at the cSAXS beamline, Swiss Light Source, Paul Scherrer Institute, Switzerland. C.D. acknowledges funding from the Leverhulme Trust (ECF-2018-016), the Isaac Newton Trust (18-08) and the L'Oréal-UNESCO UK and Ireland Fellowship For Women In Science 2019. A.H. was funded by the European Union's Horizon 2020 research and innovation programme under Marie SklodowskaCurie grant agreement number 794207 (ASIQS).

\section{Author contributions:}

C.D. and S.F. conceived the study of 3D magnetic dynamics with input from J.R.. Project discussions involved C.D., S.F., S.G., V.S, M.H., A.H., L.H., M.G-S. and J.R.. The sample fabrication was planned by C.D. and S.F. and performed by C.D., A.H., S.M. and E.M. The time-resolved 
electronics setup was designed and implemented by S.F. and J.R. The laminography endstation was designed and built by M.H. The diamond phase plate for the production of circular polarization was implemented by V.S. The synchrotron measurements were performed by C.D., S.F., M.G.-S., M.H., V.S., and J.R.. The non-magnetic laminography reconstruction code was developed by M.O., and used by C.D. with the support of M.O. The magnetic laminography reconstruction was developed and used by CD, with the support of M.G-S. Data analysis was performed by C.D. with the support of M.G-S. The time-resolved 3D magnetic data was interpreted by C.D., S.F., S.G. and M.G.-S.. C.D. wrote the manuscript with contributions from all authors. The clarity of the final manuscript was ensured by C.D., S.G., L.H. and M.G.S..

\section{Financial and Non-Financial Competing Interest Statement:}

Authors declare no competing interests.

\section{Figure captions:}

Figure 1. Time-resolved X-ray magnetic laminography. a) The experimental setup. For synchrotron X-ray magnetic laminography, ptychographic projections are measured with rightand left-handed circular polarization with the sample rotated around an axis that is at $61^{\circ}$ with respect to the $\mathrm{X}$-ray beam propagation direction. To observe the magnetization dynamics, the sample is fabricated on a Cu stripline (see magnified view in $i$, along with a scanning electron micrograph of the sample in ii), and excited with a $500 \mathrm{MHz}$ RF magnetic field produced by passing a current through the stripline. Magnetic laminography is then performed for a number of different delay times of the RF magnetic field with respect to the $\mathrm{X}$-ray pulses. $\mathrm{b}$ ) the concept of pump-probe magnetic laminography is illustrated, where the RF magnetic field excitation (red oscillatory waveform) is frequency and phase matched to the incoming $X$-ray pulses (purple waveforms). By varying the time delay $\delta$ t between the continuous RF magnetic field excitation (red) and the X-ray bunches (two temporal measurements, separated by $\delta \mathrm{t}$, are indicated by the full and dashed purple waveforms), and repeating the laminography measurement for different $\delta$ t, temporally-resolved maps of the magnetization vector field are obtained. XMCD projections of the magnetic microdisc are shown in c) and d) for the sample at $0^{\circ}$ and $45^{\circ}$, respectively. Scale bars represent $2 \mu \mathrm{m}$ in a,ii and $1 \mu \mathrm{m}$ in c, d.

Figure 2. The reconstructed three-dimensional magnetic configuration of the GdCo microdisc for zero time delay between the X-ray measurements and the RF excitation field BA. At the bottom of the disc (a), a multidomain state forms with a large central domain and two vortices. With increasing height, the vortices move towards the edges of the disc (b), until they exit the structure approximately midway through the disc thickness, resulting in a large magnetic domain in an S-state (c). At the top of the disc, the magnetic configuration is almost uniform (d). The positions of the cores of the vortices, coloured by the $y$-component of the magnetization, are shown in (e), indicating that the magnetization in the cores of the two vortices have opposite directions, i.e. opposite vortex polarizations. These vortices eventually exit through the edges of the sample approximately mid-way through its height. f) Bivariate histogram showing the number of pixels as a function of magnetization direction (angle defined with coordinate axes in a) and thickness through the sample, with the orientation of the applied 
RF field BA $\left[\theta=79^{\circ}\left(259^{\circ}\right)\right]$ indicated with purple dashed lines. g) The dominant direction of the magnetization in the central domain (black dots) and the standard deviation of $\theta_{-} m$ (red dots), which reflects the magnetization state, are given as a function of position through the thickness of the sample. Three regions of the sample are identified on the plot, the vortex state ( $i$, orange), the S-state (ii, yellow) and the single domain state (iii, blue).

Figure 3. Dynamic response of the three-dimensional magnetization at $500 \mathrm{MHz}$. a-d) The precession angle of the magnetization is plotted for the heights within the sample corresponding to Fig 2 a-d (see Fig. S4 for slice locations). In the lower part of the sample $(a, b)$, there is significant precession in the vicinity of the vortex domain walls (indicated with grey arrows in a), as well as at the edge of the sample. In the intermediate S-state (c), an enhancement of the edge modes at opposite sides of the disc is observed. In the single domain state (d), lower amplitude edge modes are present on the top left and bottom right edges of the microdisc (visualised with a colour scale that is different to that used for a-c,e,f). The threedimensional dynamics are visualised in e and $f$ by mapping the magnetisation precession: the dynamics are limited to the vortex cores (e) and edge modes, which spiral around the disc ( $f$ ). Scale bars represent $1 \mu \mathrm{m}$.

Figure 4. Domain wall dynamics in the lower region of the disc containing two vortex walls. a) The curl of the in-plane magnetization $\nabla \times m_{-} x z$ for $h=90 \mathrm{~nm}$ is shown, where the positions of the two domain walls are identified by the positive (red) and negative (blue) lines. The domain wall with positive curl in the region indicated by the dashed box is shown at different times increasing clockwise around the central image, with a reference location indicated by a dashed line. b) The dynamics of the wall with a positive curl vortex (red, vortex 1 ) are compared with the dynamics of the wall with a negative curl vortex (blue, vortex 2 ) for an example slice of height $46 \mathrm{~nm}$. The two oscillatory signals are out of phase by $3 \pi / 4 \pm \pi / 4,(0.75 \pm 0.25) \mathrm{ns}$, consistent with the breathing of the central domain with the applied RF magnetic field. Scale bar represents $1 \mu \mathrm{m}$.

\section{Data availability:}

All data associated with this manuscript will be made available on a repository when the manuscript is published.

\section{Code availability Statement:}

All analysis code and reconstruction algorithms associated with the work of this manuscript will be made available on a repository when the manuscript is published.

\section{Methods:}

\section{Experimental setup}

Laminography setup 
A gold Fresnel zone plate (FZP) with a diameter of $170 \mu \mathrm{m}$ and an outermost zone width of $60 \mathrm{~nm}$ was coherently illuminated at a photon energy of $7.247 \mathrm{keV}$, which corresponds to the maximum XMCD signal at the $\mathrm{Gd} \mathrm{L}_{3}$ edge. The FZP was fabricated with displaced zones, designed to introduce perturbations into the illumination wavefront and improve the image resolution for ptychography [37]. The FZP was used in combination with a $50 \mu$ m-diameter central stop and $30 \mu \mathrm{m}$-diameter order sorting aperture. Circular polarized light was generated by a diamond quarter wave plate phase plate positioned before the setup as presented in [18, $26]$, with a resulting degree of polarization of over $99.9 \%$, and an efficiency of approximately $60 \%$.

The sample was placed downstream of the focal spot of the FZP where the beam size reached a diameter of $5 \mu \mathrm{m}$. The sample was mounted on a 2D piezo scanner with a $100 \times 100 \mu \mathrm{m}^{2} \mathrm{scan}$ range and the scanner was installed on a rotation stage allowing $360^{\circ}$ rotation of the sample. Two linear stages with a travel range of several millimeters were used to move the rotation stage in order to keep the region of interest in the field of view at different rotation angles. The entire sample stage stack was mounted at a laminography angle of $61^{\circ}$ (angle between axis of rotation and X-ray beam propagation). This angle was chosen to provide a balance between $3 \mathrm{D}$ spatial resolution and sample absorption, and the suitability for magnetic laminography was determined using numerical simulations of magnetic laminography that are described in the next section.

Similar to previous instrumentation $[38,39]$, closed loop sample positioning and thus positioning accuracy during ptychographic scans was achieved by differential laser interferometry measuring the relative position between the sample and the beam-defining FZP in both the horizontal and vertical directions perpendicular to the $\mathrm{X}$-ray beam propagation direction [28].

The diffracted $X$-rays propagated in an evacuated flight tube and were detected with an invacuum Eiger detector [40] placed at a distance of $5.260 \mathrm{~m}$ from the sample.

\section{Time-resolved setup}

The time-resolved data were obtained with pump-probe measurements, employing the X-ray flashes generated by the synchrotron light source as a probing signal. The time structure of the Swiss Light Source is designed to provide 70 ps wide (FWHM) X-ray pulses, defining the temporal resolution of our measurements, that arrive approximately every 2 ns (repetition frequency of $499.652 \mathrm{MHz}$ ). The magnetization dynamics in the sample were excited by an RF magnetic field that was synchronized to the RF electron bunch accelerators of the synchrotron light source through an IQ modulator, which was then employed to shift the relative delay between the $\mathrm{X}$-ray pulses and the RF excitation signal.

This method locks the excitation frequency and its phase to the synchrotron repetition frequency, or its higher order harmonics. If a single-bunch filling pattern were to be employed, classical pump-probe measurements could be carried out, which would allow the investigation of different frequency ranges, or pulsed excitations.

\section{Laminography measurements}


To obtain 2D projections of the magnetization, resonant dichroic ptychography was used. The energy of the $\mathrm{X}$-rays was tuned to the absorption edge of the magnetic element in question, in this case the $L_{3}$ edge of gadolinium with an energy of $7.247 \mathrm{keV}$, and ptychographic projections were recorded with right and left circularly polarised light.

Ptychographic projections with a field of view of $7 \times 12.5 \mu \mathrm{m}^{2}$ consisted of 818 diffraction patterns with a step size of $1 \mu \mathrm{m}$ and an exposure time of $0.2 \mathrm{~s}$. The two-dimensional projections of the magnetization shown in Figure 1c,d are obtained from an average of 25 single XMCD projections, which allowed for a sufficiently high signal-to-noise ratio $2 \mathrm{D}$ image of the magnetic structure.

For magnetic laminography, ptychographic projections were measured with both right and left circularly polarised X-rays at 144 angles equally spaced over $360^{\circ}$. Within each dataset, six of the projections were blocked by the coaxial cable carrying the RF current to the sample, meaning that the laminography reconstructions were performed using 138 projections of each polarization.

The images were measured at nonsequential time delays to be sure that any observed dynamic behaviour would be independent of slight sample changes occurring over time due to heating or aging effects. The phases were measured in the following order:

$$
\pi, 0, \frac{7 \pi}{4}, \frac{3 \pi}{4}, \frac{\pi}{4}, \frac{5 \pi}{4}, \frac{\pi}{2}, \frac{3 \pi}{2}
$$

At times of $1 \mathrm{~ns}, 0 \mathrm{~ns}, 1.75 \mathrm{~ns}, 0.75 \mathrm{~ns}, 0.25 \mathrm{~ns}, 1.25 \mathrm{~ns}, 0.5 \mathrm{~ns}, 1.5 \mathrm{~ns}$, respectively.

Unfortunately, due to experimental errors it was not possible to obtain a complete reconstruction from the $\frac{\pi}{2}(0.5 \mathrm{~ns})$ laminography dataset, which is why seven time-steps are presented in the manuscript rather than eight.

Each laminography measurement consisting of 144 projections took 7 hours including overhead, meaning that each magnetic laminography measurement required 14 hours in order to measure with both left and right circular polarization. Therefore, in total, seven time steps required 4.1 days of continuous measurement and a total of 2016 projections.

\section{Laminography reconstruction (non-magnetic)}

The measured diffraction patterns were used to reconstruct the complex-valued projections $P_{n}(x, y)$. The reconstruction was performed using 300 iterations of the difference map method [41] and 300 iterations of the maximum likelihood optimization [42]. Reconstructed and unwrapped phase projections were aligned together in order to maximize their mutual consistency [43]. This is possible only because the magnetic properties of the sample do not affect the phase-based alignment procedure at the selected photon energy. Aligned and unwrapped phase projections were reconstructed by the filtered backprojection method. The laminography imaging geometry unavoidably results in missing information in the Fourier space, which is the so-called missing cone problem. Therefore, an iterative method enforcing physical constraints in the real space and measured projection in the Fourier space was used to fill the missing cone [28]. 
The non-magnetic reconstruction was used to estimate the location of the magnetic material, which is employed as a constraint in the magnetic reconstruction detailed in the following section.

\section{Magnetic Laminography}

Magnetic laminography, as well as magnetic tomography, is a special case of arbitrary projection 3D magnetic imaging. For completeness we repeat here the general formulation for arbitrary projection magnetic tomography reconstructions [29] and later show the particular application to laminography.

The amplitude of the transmissivity, $A_{n}$, measured experimentally is related to the defined XMCD projection $P_{n}$ as follows:

$$
A_{n}(x, y)=\exp \left(\frac{-2 \pi}{\lambda} P_{n}(x, y)\right)
$$

After normalization and computation of the absolute value of the transmissivity, each projection is mathematically defined in terms of rotation matrices as:

$$
P_{n}(x, y)=\frac{-r_{e}}{2 \pi} \lambda^{2}\left(\sum_{k} n_{a t}^{k} \int \Im\left\{f_{c}^{k}\left(R^{(n)} r\right)\right\} d z \pm n_{a t}^{m a g} \int \mathfrak{I}\left\{f_{m}^{(1)}\right\}\left[R^{(n) \dagger} m\left(R^{(n)} r\right)\right] \cdot \hat{z} d z\right)
$$

Where $r_{e}$ is the classical electron radius, $\lambda$ is the X-ray wavelength, $n_{a t}^{k}$ and $n_{a t}^{m a g}$ are the atomic density of the $k$ th element, and of the resonant magnetic element, respectively, and $\mathfrak{I}$ selects the imaginary part. Here, $m\left(R^{(n)} r\right)$ is the magnetization vector, and $f_{c}$ and $f_{m}^{(1)}$ are the electron density and XMCD scattering factors, respectively. The magnetic contribution originates only from the resonant element, given in the second term, whereas the electronic scattering is calculated by summing over all elements present including the magnetic element.

During the magnetic laminography reconstruction, for each iteration the reconstructed components of the magnetization from the previous iteration are used to compute the projections and compare them to the data. In this calculation, the integrals are approximated by sums, and the projection is defined by:

$$
\hat{P}_{n}(x, y)=\sum_{z}\left\{c\left[R^{(n) \dagger} m\left(R^{(n)} r\right)\right] \cdot \hat{z}+o\left(R^{(n)} r\right)\right\}
$$

Where $m$ and $o$ are the estimated magnetic and non-magnetic structures, respectively, and $c$ is a constant that relates the XMCD signal to the magnetization, including the pixel size of the dataset. At each iteration of the reconstruction, the correctness of the reconstruction is evaluated by calculating an error metric that compares the estimated dataset $\hat{P}_{n}(x, y)$ with the measured dataset $P_{n}(x, y)$. This error metric is defined as:

$$
\varepsilon=\sum_{n} \sum_{x, y}\left[\hat{P}_{n}(x, y)-P_{n}(x, y)\right]^{2}
$$

The reconstruction is then updated such that the error metric is reduced, and the reconstruction approaches the correct solution, by calculating the gradient of the error metric 
with respect to the different reconstructed variables. In this case, the gradients for the magnetization $m$ and the non-magnetic structure $o$ are analytically defined as:

$$
\begin{gathered}
\frac{\partial \varepsilon}{\partial m\left(R^{(n)} r\right)}=2 \sum_{n}\left[\hat{P}_{n}(x, y)-P_{n}(x, y)\right] R^{(n)}\left[\begin{array}{l}
0 \\
0 \\
1
\end{array}\right] \\
\frac{\partial \varepsilon}{\partial o\left(R^{(n)} r\right)}=2 \sum_{n}\left[\hat{P}_{n}(x, y)-P_{n}(x, y)\right]
\end{gathered}
$$

For magnetic laminography, the rotation matrices that define a rotation of the sample by $\varphi$ around the rotation axis, which is in turn at an angle $\theta_{L}$ with respect to the direction of propagation of the $\mathrm{X}$-rays $(\hat{Z})$, are given by:

$$
\begin{aligned}
R^{(n) \dagger} & =\left(\begin{array}{ccc}
1 & 0 & 0 \\
0 & \sin \theta_{L} & \cos \theta_{L} \\
0 & -\cos \theta_{L} & \sin \theta_{L}
\end{array}\right)\left(\begin{array}{ccc}
\cos \varphi_{n} & 0 & \sin \varphi_{n} \\
0 & 1 & 0 \\
-\sin \varphi_{n} & 0 & \cos \varphi_{n}
\end{array}\right) \\
& \left(\begin{array}{ccc}
\sin \varphi_{n} & 0 & \cos \varphi_{n} \\
-\cos \theta_{L} \sin \varphi_{n} & \sin \theta_{L} & \cos \theta_{L} \cos \varphi_{n} \\
-\sin \theta_{L} \sin \varphi_{n} & -\cos \theta_{L} & \sin \theta_{L} \cos \varphi_{n}
\end{array}\right)
\end{aligned}
$$

As a result, the gradients for the components of the magnetization are defined as:

$$
\frac{\partial \varepsilon}{\partial m\left(R^{(n)} r\right)}=2 \sum_{n}\left[P_{n}(x, y)-P_{n}(x, y)\right]\left[\begin{array}{c}
-\sin \theta_{L} \sin \varphi_{n} \\
-\cos \theta_{L} \\
\sin \theta_{L} \cos \varphi_{n}
\end{array}\right]
$$

For $0<\theta_{L}<90^{\circ}$, all three components of the gradient are non-zero, meaning that the measured projections are sensitive to, and contain information about, all three components of the magnetization. This in turn suggests that they can in principle be recovered with a dataset measured around a single rotation axis. To determine whether this is the case, we performed numerical simulations of magnetic laminography for a complex 3D magnetic structure containing vortices, domain walls and Bloch points, as in [18]. For the simulations, 360 XMCD projections were measured around the laminography rotation axis and, in order to reconstruct the magnetic signal, 50 iterations of the arbitrary projection magnetic reconstruction algorithm (APMRA) were used. The laminography axis was varied between $0^{\circ}$ and $90^{\circ}$ to determine the optimal geometry for determining the 3D magnetization vector field with magnetic laminography. 


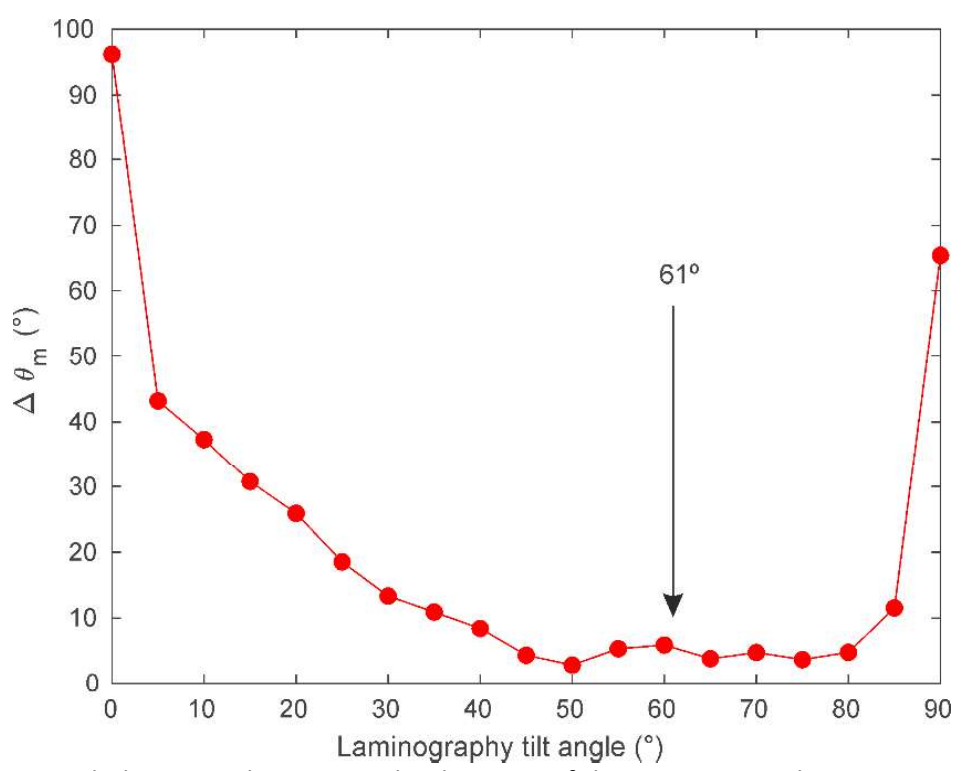

Extended Data 1: The error in the direction of the reconstructed magnetization vector field $\left(\Delta \theta_{m}\right)$ for different laminography tilt angles is determined by numerical simulations of magnetic laminography. The simulations show that there exists a range of angles $\left(30^{\circ}-80^{\circ}\right)$ for which the 3D structure can be reconstructed with a high degree of accuracy. The accuracy of the reconstruction is determined by calculating the RMS error of the direction of the reconstructed magnetization with respect to the original structure $\left(\Delta \theta_{m}\right)$. The tilt angle used in the experiment presented in this manuscript is $61^{\circ}$, which lies comfortably within this range and is indicated with an arrow.

The error in the reconstructed magnetic structure with respect to the original magnetic structure was defined as the error in the local magnetization 3D orientation and was calculated for a range of laminography tilt angles. This error in the direction of the reconstructed magnetization direction is shown in Extended Data 1. It can be seen that a reasonably wide range of angles exists for which the 3D magnetic structure is reconstructed with a high degree of accuracy. In particular, for the angle used in this work $\theta_{L}=61.2^{\circ}, 99 \%$ of all voxels have an error less than $6^{\circ}$ of the direction of the magnetization in three dimensions.

\section{Magnetic laminography reconstruction}

The three-dimensional magnetic structure was reconstructed using 10 iterations of the GPUimplemented version of the gradient-based arbitrary projection reconstruction code described in [29] and made available at [44], using the rotation matrices described above. During the magnetic reconstruction, the magnetization was constrained to the magnetic material using a mask calculated from the non-magnetic laminography reconstruction. No additional regularisation of the magnetization was implemented.

Once reconstructed, the three components of the magnetization were filtered using a Hanning filter with a cutoff spatial frequency of $1 / 6$ with respect to the maximum spatial frequency in order to remove high frequency noise, and the magnetization was then normalised by its magnitude to obtain a magnetic vector field of uniform amplitude.

The magnetic reconstruction for each time-step was performed independently using the same reconstruction parameters.

\section{Spatial resolution and accuracy of magnetic reconstruction}


The half-period spatial resolution of the magnetic reconstruction was estimated by taking a line profile of the $m_{x}$ and $m_{z}$ components across the vortex domain wall and measuring the $25-75 \%$ edge sharpness [45], as shown in Extended Data 2, which was found to be $50 \mathrm{~nm}$.

Micromagnetic simulations show that, at the surface of a sample, the width of vortex core is of the order of 20-30 nm, which increases rapidly as one moves into the bulk of the disc.

Considering that the measured edge sharpness is a convolution of the size of the vortex core and the spatial resolution, we therefore estimate the half-period spatial resolution of the magnetic reconstruction to be approximately $50 \mathrm{~nm}$.
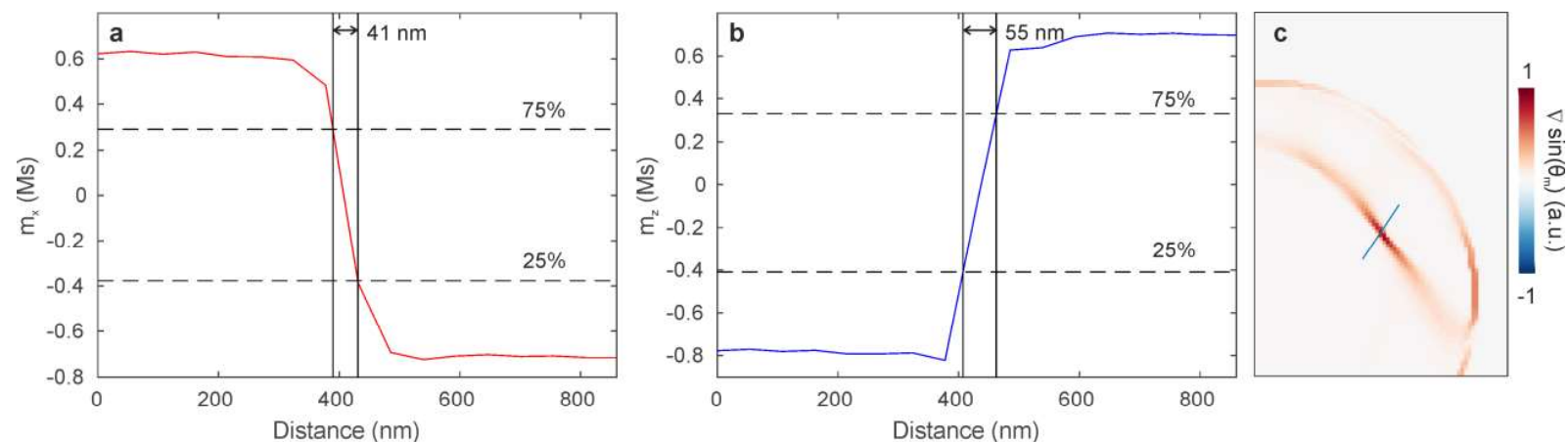

Extended Data 2: Line profiles of (a) the $m_{x}$ and (b) the $m_{z}$ components of the magnetization across the vortex domain wall. The $25 \%-75 \%$ edge sharpness is measured to be on average $50 \mathrm{~nm}$, indicating a half-period spatial resolution of $50 \mathrm{~nm}$. c) The line along which the profile is measured is drawn in blue

A further estimate of the half-period spatial resolution was also obtained by calculating the Fourier Shell Correlation (FSC) of the three components of the magnetization and the in-plane vector fields in the three Cartesian planes, shown in Extended Data 3. The FSC indicates an average spatial resolution of $184 \mathrm{~nm}, 360 \mathrm{~nm}$ and $180 \mathrm{~nm}$ for the $m_{x}, m_{y}$ and $m_{z}$ components respectively. The difference between the edge sharpness and FSC estimated values for the resolution can be explained by the spatial sparsity of the data, i.e. the small number of features with high spatial frequencies present in the sample, which leads to underestimation of the spatial resolution based on FSC [46].
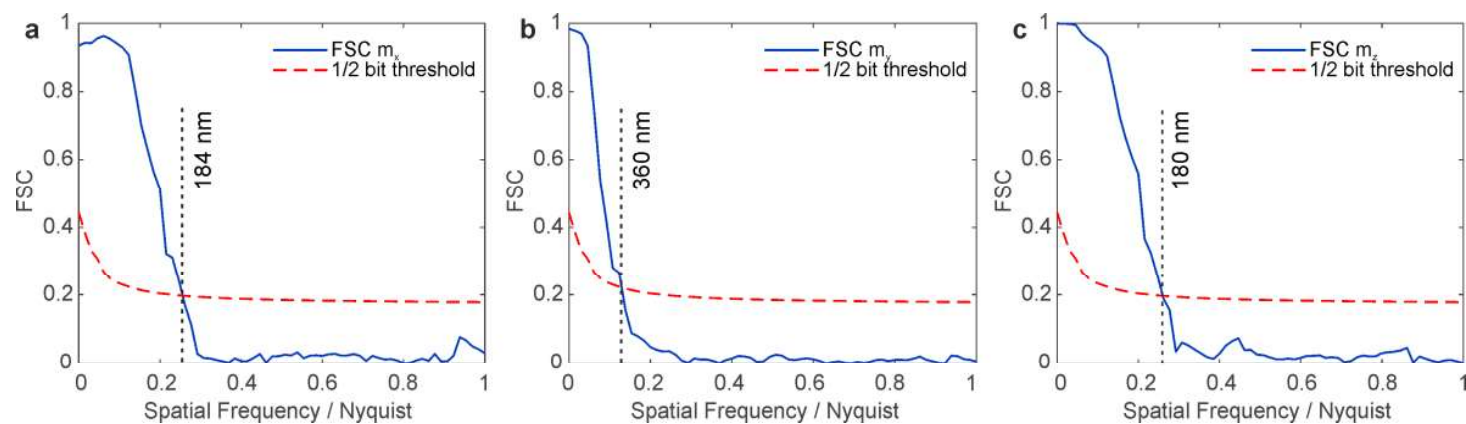

Extended Data 3. Fourier Shell Correlation of (a) the $m_{x}$, (b) the $m_{y}$ and (c) the $m_{z}$ components of the magnetization. An estimate of the spatial resolution is obtained using the $1 / 2$ bit threshold [36] (red dashed line) and is found to be $184 \mathrm{~nm}, 360 \mathrm{~nm}$ and $180 \mathrm{~nm}$ for the $m_{x}, m_{y}$ and $m_{z}$ components of the magnetization, respectively

In the time-resolved measurement, we are able to track displacements of the vortex domain wall with an accuracy significantly higher than the spatial resolution. In particular, we track 
oscillations in the domain wall location with an amplitude of $45 \mathrm{~nm}$ and $74 \mathrm{~nm}$ for the vortex domain walls with negative and positive vorticity, respectively. This sub-spatial resolution tracking of the domain wall is possible as it is dependent on the signal-to-noise ratio of the reconstruction, and not the global or local spatial resolution.

\section{Three-dimensional magnetic structure}

For reference, the heights for which the magnetization is shown in Figure 2a-d, and the 500 $\mathrm{MHz}$ modes in Figure 3a-d, are indicated on the three-dimensional rendering of the $500 \mathrm{MHz}$ modes in Extended Data 4.

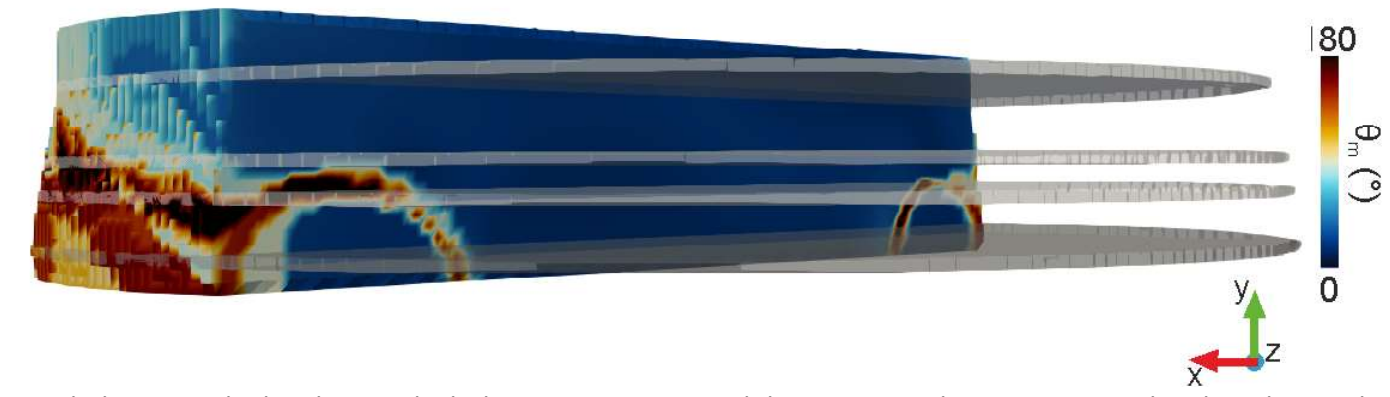

Extended Data 4. The heights at which the magnetization and dynamics are shown in Figs 2a-d and 3a-d are indicated by semitransparent discs, superimposed on the three-dimensional rendering of the magnetization modes shown in Figure $3 \mathrm{e}$.

\section{Data Analysis: Calculation of vortex domain wall displacement}

The displacements of the core of the vortex domain walls were calculated using two different procedures that both make use of the sub-pixel image registration algorithm described in [47]:

Vortex-tracking based on $\nabla \theta_{m}$ (vortex 1$)$ :

In order to track the displacement of the core of vortex 1 , the divergence of the direction of the in-plane magnetization was calculated for a region of the sample surrounding the vortex core of the domain wall. As an example, the divergence of $\theta_{m}$ is shown in Extended Data 5 where, at the cores of the vortices, there is a sharp peak in the gradient of $\theta_{m}$ which has a FWHM of only a few pixels. The location of the vortex core for each time-step was determined by aligning the image of $\left[\nabla \sin \left(\theta_{m}\right)\right]_{t}$ with a reference two-dimensional Gaussian with a FWHM of 1 pixel (46.8 $\mathrm{nm})$. The same calculation of the vortex core displacement was performed for all slices of the three-dimensional magnetic structure in which the vortex domain wall was present, and the motion of the core of the vortex domain wall as a function of height was determined.

Vortex-tracking based on $\sin \left(\theta_{m}\right)$ (vortex 2):

While the determination of $\nabla \theta_{m}$ worked well for tracking the vortex core with positive curl in the plane of the slice (vortex 1 ), shown in Figure 4, when applied to the second vortex domain wall with negative vorticity, the tracking was found to be more susceptible to error due to the more complex structure of the core of the domain wall. To reduce this error, we instead aligned images with a signal that was not limited to the vortex core as seen in Extended Data $5 \mathrm{a}$, but was present throughout the images (Extended Data $5 b$ ). For this, we calculated $\sin \left(\theta_{m}\right)$, shown in Extended Data $5 b$, which had a non-zero signal in the majority of the image and is a 
continuous function that avoids the effect of phase wrapping that manifests in the sharp transition of the angle from $0 \rightarrow 2 \pi$.

The components of the motion of the vortex cores parallel and perpendicular to the long axes of the domain walls were calculated and compared. In general, for both domain walls, the motion perpendicular to the long axis of the domain wall could be identified as oscillatory. However, for the motion parallel to the long axis of the domain wall, no clear oscillatory motion could be determined. The challenges in determining the motion of the vortex cores parallel to the long axes of the domain walls are likely to be due to the extreme ellipticity of the vortex cores, that is due in turn to the anisotropy in the system. In this case, it is likely that the much smaller displacement of the cores parallel to the long axis of the domain wall is below the current accuracy of this measurement.
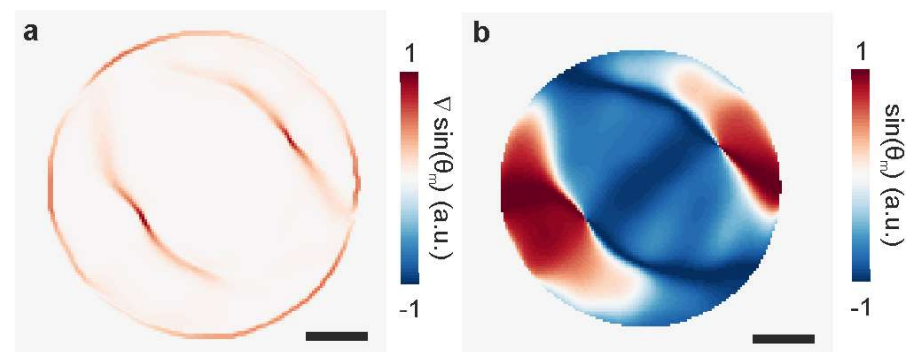

Extended Data 5. Images of a) $\nabla \sin \left(\theta_{m}\right)$ and b) $\sin \left(\theta_{m}\right)$ of the lowest slice of the magnetization. One can see that for $\nabla \sin \left(\theta_{m}\right)$ in a), the signal is limited to the domain walls, whereas for $\sin \left(\theta_{m}\right)$ the signal is non-zero throughout the majority of the structure.

The motion of the first and second vortex domain walls (vortices 1 and 2 in Figure 4a) in the direction perpendicular to their long axes, indicated by a pair of grey arrows in Extended Data 6 for each vortex, are given as a function of height in Extended Data $6 a$ and $b$, respectively. Here one can identify a similar periodic temporal behaviour to the first vortex domain wall shown in Figure 4, albeit with a smaller amplitude of $(45 \pm 13) \mathrm{nm}$, and a phase shift of $(0.75 \pm 0.25) \mathrm{ns}$ with respect to the first vortex domain wall. 

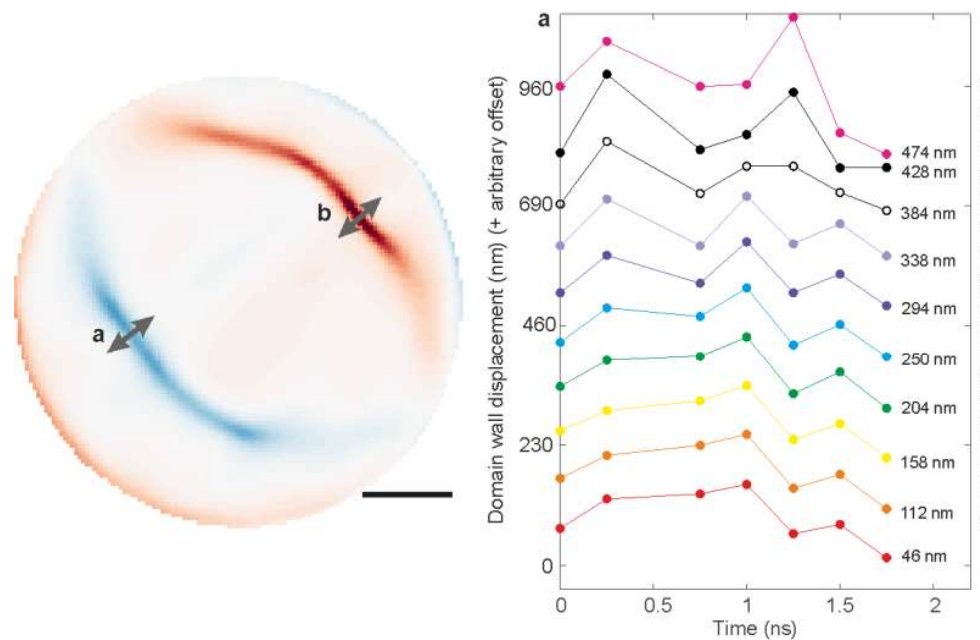

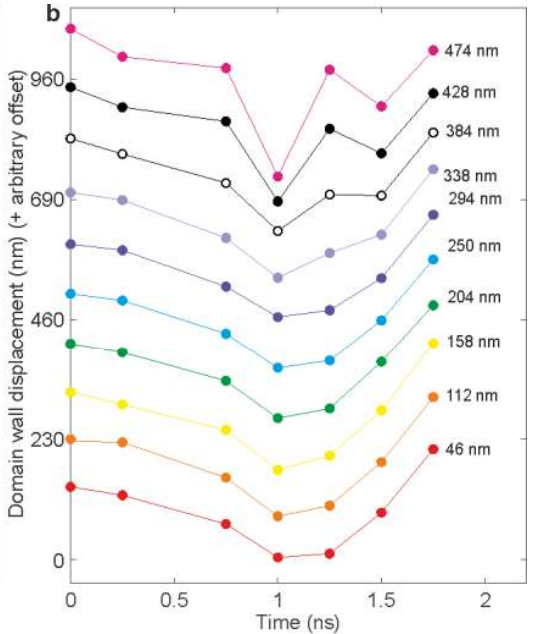

Extended Data 6. Displacement of the vortex domain walls with negative vorticity (a, blue in Figure 4 ) and positive vorticity (b, red in Figure 4) in the direction perpendicular to the long axis of the domain wall (indicated by grey arrows) as a function of time for different heights within the structure. The oscillatory behaviour is clearest for both vortices at a height of $46 \mathrm{~nm}$ and is less clear at the top. When comparing the motion of the two vortex domain walls, the oscillations appear to be consistently out of phase through the height of the sample. Scale bar is $1 \mu \mathrm{m}$.

\section{References for the Methods section:}

37. Odstrčil, M., et al., Towards optimized illumination for high-resolution ptychography. Optics Express, 2019. 27(10): p. 14981-14997.

38. Holler, M. and Raabe, J., Error motion compensating tracking interferometer for the position measurement of objects with rotational degree of freedom. Vol. 54. 2015: SPIE. 1-7, 7.

39. Holler, M., et al., OMNY - A tOMography Nano crYo stage. Review of Scientific Instruments, 2018. 89(4): p. 043706.

40. Guizar-Sicairos, M., et al., High-throughput ptychography using Eiger: scanning X-ray nano-imaging of extended regions. Optics Express, 2014. 22(12): p. 14859-14870.

41. Thibault, P., et al., High-resolution scanning x-ray diffraction microscopy. Science, 2008. 321(5887): $\mathrm{p}$. 379-382.

42. Thibault, P. and Guizar-Sicairos, M., Maximum-likelihood refinement for coherent diffractive imaging. New Journal of Physics, 2012. 14: p. 063004.

43. Odstrčil, M., et al., Alignment methods for nanotomography with deep subpixel accuracy. Optics Express, 2019. 27(25): p. 36637-36652.

44. Donnelly, C. and Guizar-Sicairos, M., Magnetic tomography reconstruction algorithm 2018: http://doi.org/10.5281/zenodo.1324335.

45. Attwood, D., Soft X-Rays and Extreme Ultraviolet Radiation: Chapter 9. 1999: Cambridge University Press.

46. van Heel, M. and Schatz, M., Fourier shell correlation threshold criteria. Journal of Structural Biology, 2005. 151(3): p. 250-262.

47. Guizar-Sicairos, M., Thurman, S.T. and Fienup, J.R., Efficient subpixel image registration algorithms. Optics Letters, 2008. 33(2): p. 156-158. 